\begin{tabular}{|c|l|}
\hline Title & NMR Study of the Dimerized State in Cul r2S4 \\
\hline Author(s) & Kumagai, Ken-ichi; Sasaki, Mayumi; Kakuy anagi, Kosuke; Nagata, Shoichi \\
\hline Citation & $\begin{array}{l}\text { Hyperfine Interactions, 159(1-4), 181-185 } \\
\text { https://doi.org/10.1007/310751-005-90943 }\end{array}$ \\
\hline Issue Date & $2005-12$ \\
\hline Doc URL & http://hdl.handle.net/2115/8373 \\
\hline Rights & The original publication is available at www.springerlink.com \\
\hline Type & article (author version) \\
\hline File Information & NQI 2004spinelmuscriptprer.pdf \\
\hline
\end{tabular}

Instructions for use 


\title{
NMR Study of the Dimerized State in $\mathrm{CuIr}_{2} \mathrm{~S}_{4}$
}

\author{
Ken-ichi Kumagai ${ }^{1 *}$, Mayumi Sasaki ${ }^{1}$, Kosuke Kakuyanagi ${ }^{1}$, Shoichi Nagata ${ }^{2}$ \\ ${ }^{1}$ Division of Physics, Graduate School of Science, Hokkaido University, \\ Sapporo 060-0081, Japan, ${ }^{2}$ Department of Materials Science and Engineering, \\ Muroran Institute of Technology, Muroran 050-8585, Japan
}

(Dated: March 31, 2006)

\begin{abstract}
We have investigated the metal-insulator transition (MIT) of $\mathrm{CuIr}_{2} \mathrm{~S}_{4}$ by a high resolution NMR measurement. The $\mathrm{Cu}-\mathrm{NMR}$ spectrum below $T_{M I}$ is broadened and split into four $\mathrm{Cu}$ signals with sizable electric quadrupole interactions. The NMR results are consistent with the charge ordering of $\operatorname{Ir}^{3+}$ and $\operatorname{Ir}^{4+}$ and the spin dimerization of $\operatorname{Ir}^{4+}$ spins, as revealed by a recent $X$-ray study.

keywords: NMR, metal-insulator transition, spinel, $\mathrm{CuIr}_{2} \mathrm{~S}_{4}$
\end{abstract}

PACS numbers:

\section{INTRODUCTION}

In calcogenide spinel $\mathrm{CuIr}_{2} \mathrm{~S}_{4}$, the metal-insulator transition (MIT) at $T_{M I} \sim 230 \mathrm{~K}[1]$ has attracted much attention. Since NMR [2] and photoemission [3] measurements show that the $\mathrm{Cu}$ ions are monovalent in the insulating state and thus the nominal valence of the Ir atoms is 3.5 , the nonmagnetic ground state below $T_{M I}$ is puzzling for possible $\operatorname{Ir}^{3+}(S=0)$ and $\operatorname{Ir}^{4+}(S=1 / 2)$ configurations. There are discussions about the charge order of $\operatorname{Ir}^{3+}(S=0)$ and $\operatorname{Ir}^{4+}(S=1 / 2)$ ions, and consequently possible spin singlet dimers in the insulating state. This MIT is accompanied by a structural transition from cubic to tetragonal (triclinic) symmetry with volume contraction of $0.7 \%$. [4] Recently, a precise $X$-ray measurement [5] has shown that a charge ordering of $\mathrm{Ir}^{3+}$ and $\mathrm{Ir}^{4+}$ and spin dimerization of $\operatorname{Ir}^{4+}$ ions occurs simultaneously below $T_{M I}$. The Ir sublattice consists of two type of Ir bicapped hexagonal rings which described as $\mathrm{Ir}^{3+}$ and $\mathrm{Ir}^{4+}$ octamers. In order to elucidate the origin of the MIT in the $\mathrm{Cu}$-spinels, it is important to accumulate the information about the valence state and magnetic properties in the insulating state from a microscopic point of view. Here, we investigate the evolution of the electronic state associated with MIT of $\mathrm{CuIr}_{2} \mathrm{~S}_{4}$ by a Cu-NMR study using a high resolution NMR spectroscopy.

\section{EXPERIMENTAL}

Polycrystalline samples were prepared by solid state reaction [1]. NMR was measured by the conventional phase coherent pulse method with a highly-homogeneous superconducting magnet. Spectra of ${ }^{63} \mathrm{Cu}$ were obtained by the Fourier Transformed-NMR with a constant magnetic field $(H=9.4 \mathrm{~T})$.

\section{RESULTS AND DISCUSSION}

As shown in Fig. 1. we obtained very narrow ${ }^{63} \mathrm{Cu}$ spectra above $T_{M I}$ in $\mathrm{CuIr}_{2} \mathrm{~S}_{4}$. The line width is less than $5 \mathrm{kHz}$ without any notable anisotropic Knight shift and nuclear quadrupole interactions. The ${ }^{63} \mathrm{Cu}$ NMR spectrum becomes broad below $T_{M I}$, and shows several peaks within the narrow range of $100 \mathrm{kHz}$. As the MIT is of a first order transition [1], NMR signals in the metallic and insulating phase coexist for between $230 \mathrm{~K}$ and $220 \mathrm{~K}$. The isotropic part of the Knight shift of $\sim-0.08 \%$ at the metallic state indicates that there exists a negative contribution of the Knight shift from core polarizations of the $\mathrm{Cu} 3 d$ electrons. Below $T_{M I}$, the opening of a band gap reduces the Knight shift. The absence of apparent temperature dependence of the Knight shift below $T_{M I}$ indicates that the electronic state is basically nonmagnetic in the insulating state, suggesting that both $\mathrm{Cu}$ and the $\mathrm{Ir}$ atoms in $\mathrm{CuIr}_{2} \mathrm{~S}_{4}$ are in nonmagnetic states.

Because of the lattice distortion from cubic to triclinic symmetry below $T_{M I}$, an appreciable electric field gradient (EFG) at the $\mathrm{Cu}$ site is expected. Thus, the nuclear quadrupole interaction for the $\mathrm{Cu}$ nuclei $(I=3 / 2)$ is not negligible below $T_{M I}$. We have calculated the EFG at the $\mathrm{Cu}$ site with a point charge model. For this purpose, we use atomic positions and ionic configurations of $\mathrm{Ir}^{3+}$ and $\mathrm{Ir}^{4+}$ obtained by the recent precise $X$ - ray measurement. [6] The calculation reveals that only one equivalent $\mathrm{Cu}$ site in the cubic symmetry above $T_{M I}$ changes to 4 non equivalent $\mathrm{Cu}$ sites. The calculated parameters of $V_{z z}$, and $\eta$ are given in Table 1 . The number of the $\mathrm{Cu}$ sites correspond to the ones assigned in [5].

We simulate the split spectrum by taking into consideration the perturbation of the nuclear quadrupole interaction for the Zeeman field. The best fitting is achieved with superposition of 4 components of the spectrum with the slightly-different Knight shift as shown in Fig. 2. The NMR parameters for the best fittings are listed in Table 2. From the differences of the quadrupole frequency, $\nu_{Q}$, and anisotropy parameter, $\eta$, we assign each NMR component to the signal from the $\mathrm{Cu}$ atoms defined in [5]. As seen in Fig. 2, the fitting to the experimental data is satisfactory. This indicates that the charge separation and the possible dimerization of $\operatorname{Ir}^{4+}$ proposed by $X$-ray study are reasonably confirmed by the NMR study at a microscopic point view. 


\begin{tabular}{|c|c|c|c|c|}
\hline & $\mathrm{Cu}(1)$ & $\mathrm{Cu}(2$ & $\mathrm{Cu}(3$ & \\
\hline & & & & \\
\hline$c a l$ & & 0.85 & 0.74 & \\
\hline
\end{tabular}

TABLE I: Calculated EFG parameters of insulating state in $\mathrm{CuIr}_{2} \mathrm{~S}_{4}$.

\begin{tabular}{|c|c|c|c|c|}
\hline & line(1) & line $(2)$ & line $(3)$ & line $(4)$ \\
\hline$K^{\text {iso }}(\%)$ & 0.12 & 0.104 & 0.09 & 0.13 \\
\hline$\nu_{Q}(\mathrm{kHz})$ & 88 & 26 & 14 & 89 \\
\hline$\eta$ & 0.34 & 0.86 & 0.74 & 0.92 \\
\hline
\end{tabular}

TABLE II: Fitting parameters of NMR shift and nuclear quadrupole interactions.

Finally, we show the nuclear spin lattice relaxation time, $T_{1}$, of ${ }^{63} \mathrm{Cu}$ in Fig. 3. At the metallic state above $T_{M I},\left(T_{1} T\right)^{-1}$ obeys the Korringa relation (temperatureindependent), and decreases largely at $T_{M I}$ due to the opening of the band gap in the insulating state. An in- teresting feature is that the temperature dependence of $\left(T_{1} T\right)^{-1}$ obeys a power law (the $T^{2}$-relation) and not a thermally activated (exponential relation) behavior. This result indicates that the band gap in the insulating state of $\mathrm{CuIr}_{2} \mathrm{~S}_{4}$ is quite anisotropic or that spin fluctuations are in very unusual nature.

\section{SUMMARY}

We have investigated ${ }^{63} \mathrm{Cu}-\mathrm{NMR}$ in $\mathrm{CuIr}_{2} \mathrm{~S}_{4} \cdot{ }^{63} \mathrm{Cu}-$ NMR spectrum consists of 4 components of non equivalent $\mathrm{Cu}-\mathrm{NMR}$ signals with diffrerent Knight shift and nuclear quadrupole interaction in the insulating state. The charge ordering of the $\mathrm{Ir}^{3+}$ and $\mathrm{Ir}^{4+}$ and the dimerization of $\mathrm{Ir}^{4+}$ spins in $\mathrm{CuIr}_{2} \mathrm{~S}_{4}$ are verified by the NMR study.

The authors would like to thank for Dr. Y. Horibe for fruitful discussions and kind informing the atomic coordination.
[1] S. Nagata, T. Hagino, Y. Seki, T. Bitoh, Physica B194196 (1994) 1077, T. Hagino, T. Tojo, T. Atake and S. Nagata, Philos. Mag. B71 (1995) 881.

[2] K. Kumagai, S. Tsuji, T. Hagino, S. Nagata, Spectroscopy of Mott Insulator and Correlated Metals, eds. by A. Fujimori and Y. Tokura, (Springer-Verlag, 1995) p255.

[3] J. Matsuno, T. Mizokawa, A. Fujimori, D.A. Zatsepin, V.R. Galakhov, E.Z. Kurmaev, Y. Kato, S. Nagata,, Phys.
Rev. B55 (1997) R15979.

[4] T. Furubayashi, T. Matsumoto, T. Hagino and S. Nagata, J. Phys. Soc. Jpn. 63 (1994) 3333.

[5] P. G. Radaelli, Y. Horibe, M.J. Gutmann, H. Ishibashi, C.H. Chen, R.M. Ibberson, Y. Koyama, Y. S. Hor, V. Kiryukhin, S.W. Cheong, Nature 416 (2002) 155.

[6] Y. Horibe, Private communication. 


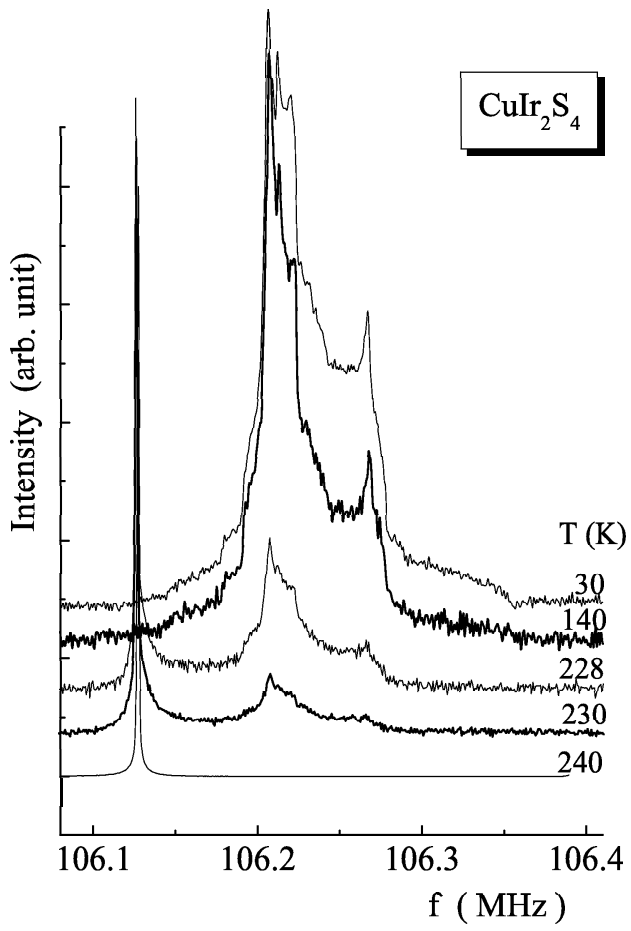

FIG. 1: ${ }^{63} \mathrm{Cu}-\mathrm{NMR}$ spectra of $\mathrm{CuIr}_{2} \mathrm{~S}_{4}$ at various temperatures. The spectra at $T=230 \mathrm{~K}$ and $T=140 \mathrm{~K}$ are shown by bold lines.

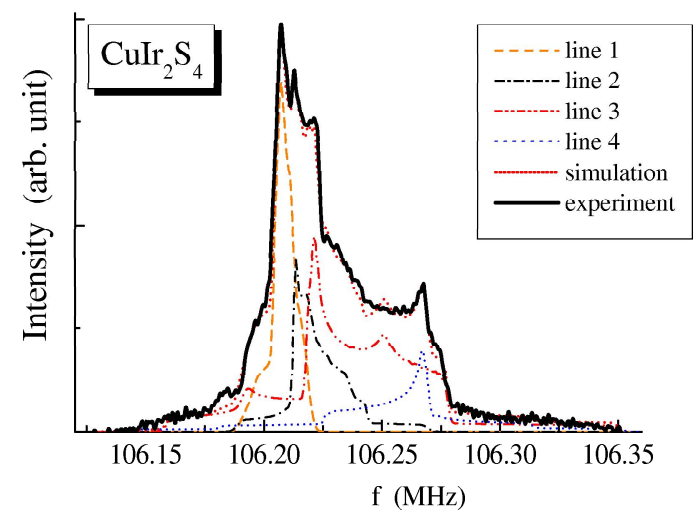

FIG. 2: ${ }^{63} \mathrm{Cu}-\mathrm{NMR}$ spectrum of $\mathrm{CuIr}_{2} \mathrm{~S}_{4}$ at $T=100 \mathrm{~K}$. Simulated spectrum which is summed up with NMR lines with reasonable values of quadrupole frequency and Knight shift for inequivalent $\mathrm{Cu}$ site are shown.

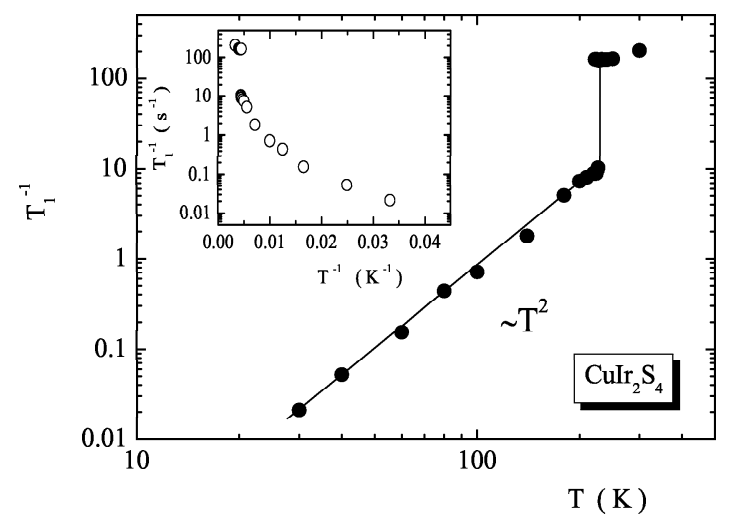

FIG. 3: Temperature dependence of nuclear spin-lattice relaxation rate, $\left(T_{1} T\right)^{-1}$ in $\mathrm{Culr}_{2} \mathrm{~S}_{4}$. The inset shows the $T_{1}^{-1}$ as a function of $T^{-1}$. 